OPEN ACCESS

Edited by:

Yi-Wei Tang,

Cepheid, United States

Reviewed by:

Zongde Zhang,

Capital Medical University, China Charles William Stratton, Vanderbilt University Medical Center, United States

${ }^{*}$ Correspondence:

Jia-Tang Zhang zjt1128@aliyun.com

Specialty section:

This article was submitted to Clinical Microbiology,

a section of the journal

Frontiers in Cellular and Infection

Microbiology

Received: 29 November 2019 Accepted: 19 February 2020

Published: 05 March 2020

Citation:

Xing X-W, Zhang J-T, Ma Y-B, He $M-W$, Yao G-E, Wang W, Qi X-K, Chen $X-Y, W u L$, Wang $X-L$, Huang $Y-H, D u$

$J$, Wang $H-F$, Wang $R-F$, Yang $F$ and

Yu S-Y (2020) Metagenomic

Next-Generation Sequencing for Diagnosis of Infectious Encephalitis and Meningitis: A Large, Prospective

Case Series of 213 Patients.

Front. Cell. Infect. Microbiol. 10:88

doi: $10.3389 / f c i m b .2020 .00088$

\section{Metagenomic Next-Generation Sequencing for Diagnosis of Infectious Encephalitis and Meningitis: A Large, Prospective Case Series of 213 Patients}

\author{
Xiao-Wei Xing ${ }^{1}$, Jia-Tang Zhang ${ }^{2,3 *}$, Yu-Bao $\mathrm{Ma}^{2}$, Mian-Wang $\mathrm{He}^{2}$, Guo-En Yao ${ }^{4}$, \\ Wei Wang ${ }^{5}$, Xiao-Kun Qi ${ }^{6}$, Xiao-Yan Chen ${ }^{2}$, Lei Wu ${ }^{2}$, Xiao-Lin Wang ${ }^{2}$, Yong-Hua Huang ${ }^{7}$, \\ Juan $\mathrm{Du}^{8}$, Hong-Fen Wang ${ }^{2}$, Rong-Fei Wang ${ }^{2}$, Fei Yang ${ }^{2}$ and Sheng-Yuan $\mathrm{Yu}^{2,3}$ \\ ${ }^{1}$ Department of Neurology, Hainan Hospital of PLA General Hospital, Sanya, China, ${ }^{2}$ Department of Neurology, First Medical \\ Center of PLA General Hospital, Beijing, China, ${ }^{3}$ Medical School of Chinese PLA, Beijing, China, ${ }^{4}$ Department of Neurology, \\ Fourth Medical Center of PLA General Hospital, Beijing, China, ${ }^{5}$ Department of Neurology, Eighth Medical Center of PLA \\ General Hospital, Beijing, China, ${ }^{6}$ Department of Neurology, Sixth Medical Center of PLA General Hospital, Beijing, China, \\ ${ }^{7}$ Department of Neurology, Seventh Medical Center of PLA General Hospital, Beijing, China, ${ }^{8}$ Department of Neurology, PLA \\ Strategic Support Force Characteristic Medical Center, Beijing, China
}

Purpose: We assessed the performance of metagenomic next-generation sequencing (mNGS) in the diagnosis of infectious encephalitis and meningitis.

Methods: This was a prospective multicenter study. Cerebrospinal fluid samples from patients with viral encephalitis and/or meningitis, tuberculous meningitis, bacterial meningitis, fungal meningitis, and non-central nervous system (CNS) infections were subjected to mNGS.

Results: In total, 213 patients with infectious and non-infectious CNS diseases were finally enrolled from November 2016 to May 2019; the mNGS-positive detection rate of definite CNS infections was $57.0 \%$. At a species-specific read number (SSRN) $\geq 2$, mNGS performance in the diagnosis of definite viral encephalitis and/or meningitis was optimal (area under the curve $[\mathrm{AUC}]=0.659,95 \%$ confidence interval $[\mathrm{Cl}]=$ $0.566-0.751$ ); the positivity rate was $42.6 \%$. At a genus-specific read number $\geq 1, \mathrm{mNGS}$ performance in the diagnosis of tuberculous meningitis (definite or probable) was optimal $(A \cup C=0.619,95 \% \mathrm{Cl}=0.516-0.721)$; the positivity rate was $27.3 \%$. At $S S R N s \geq 5$ or 10 , the diagnostic performance was optimal for definite bacterial meningitis ( $A \cup C=0.846$, $95 \% \mathrm{Cl}=0.711-0.981$ ); the sensitivity was $73.3 \%$. The sensitivities of mNGS (at SSRN $\geq 2$ ) in the diagnosis of cryptococcal meningitis and cerebral aspergillosis were 76.92 and $80 \%$, respectively.

Conclusion: mNGS of cerebrospinal fluid effectively identifies pathogens causing infectious CNS diseases. mNGS should be used in conjunction with conventional microbiological testing.

Trial Registration: Chinese Clinical Trial Registry, ChiCTR1800020442.

Keywords: encephalitis, meningitis, pathogens, metagenomic next-generation sequencing, diagnosis 


\section{INTRODUCTION}

Infectious encephalitis and meningitis are severe clinical conditions associated with high rates of morbidity and mortality worldwide (Venkatesan et al., 2013). However, the specific pathogens are not identified in $>50 \%$ of patients with acute encephalitis cases (Glaser et al., 2006) because the volumes of available cerebrospinal fluid (CSF) may be low, and the blood-brain barrier causes pathogens to be retained in the brain. Conventional microbiological tests (smears, culture, immunological tests, and polymerase chain reaction) often fail to detect pathogens that cause encephalitis and meningitis. If early tests do not accurately identify the pathogen, treatment is often inappropriate, potentially triggering severe complications or death. Viral encephalitis and meningitis, tuberculous meningitis (TBM), bacterial meningitis, and fungal meningitis constitute most cases of infectious encephalitis and meningitis. However, these four types of infections may exhibit similar clinical manifestations and CSF findings (e.g., intracranial pressures, as well as white blood cell, glucose, and protein levels). Compared with the traditional method, there is lack of a reliable approach for the simultaneous identification of microorganisms, including various viruses, bacteria, fungi, etc.

Metagenomic next-generation sequencing (mNGS), a novel and promising approach, allows simultaneous and unbiased identification of all microorganisms in human samples (Goldberg et al., 2015; Forbes et al., 2017). Some studies have used mNGS to diagnose infectious central nervous system (CNS) diseases, but most of them included small sample sizes and were focused on specific viruses, bacteria, fungi or prokaryotes (Wilson et al., 2014; Guan et al., 2016; Yao et al., 2016; Xing et al., 2019). Recently, a somewhat larger study (58 patients) found that mNGS improved the diagnosis of neurological infections, but the mNGS-positivity rate was only 22\% (Wilson et al., 2019). Thus, the performance of mNGS in diagnosis of infectious encephalitis and meningitis should be studied using a larger sample. Furthermore, because microbial genome sizes and lifestyles differ, interpretation of mNGS data requires careful analysis. Thus, we performed a large prospective study to evaluate mNGS performance in the diagnosis of infectious encephalitis and meningitis.

\section{METHODS}

\section{Study Design}

This prospective multicenter study used clinical information to diagnose infectious encephalitis and/or meningitis in six teaching hospitals in Beijing, China. The inclusion criteria were as follows: high-level clinical suspicion of CNS infectious diseases; and lumbar puncture in uninfected patients. The exclusion criteria were as follows: refusal to undergo lumbar puncture; any contraindication for such puncture; and a diagnosis of autoimmune encephalitis. Eligible patients were divided into five groups according to their final diagnoses: viral encephalitis and/or meningitis, TBM, bacterial meningitis, fungal meningitis, and CNS non-infection. We included patients with definite or probable diagnoses of CNS viral infections (Jeffery et al.,
1997), TBM (Marais et al., 2010) and bacterial meningitis (Mudaliar et al., 2006). Fungal meningitis was confirmed via conventional microbiological testing. Cryptococcal meningitis was confirmed via India ink staining or fungal culture, as well as histopathological evidence of cerebral aspergillosis. All patients had undergone at least 3 months of follow-up. Figure 1 shows the flow chart of study enrolment.

\section{mNGS of CSF}

CSF specimens were collected in accordance with standard aseptic procedures, snap frozen, stored at $-20^{\circ} \mathrm{C}$, and subjected to mNGS within $24 \mathrm{~h}$. The DNA of blood samples collected from healthy volunteers was fragmented and mixed with water in a certain proportions (negative controls). Glass beads were added to CSF samples, followed by vigorous agitation; DNA extraction; and DNA library construction. Quality-controlled libraries were sequenced on a BGISEQ-500/50 platform (BGI-Tianjin, Tianjin, China); an average of 20 million reads was obtained for each sample. After removal of human sequences, the remaining data were aligned to bacterial, virus, fungal and protozoan databases, as described in detail elsewhere (Xing et al., 2018, 2019).

\section{Interpretation of mNGS Data}

A final sequencing list of suspected pathogenic microorganisms was obtained after removal of common background microorganisms and those that had appeared in $>50 \%$ of samples during the past 3 months, compared to the negative controls. Next, the sequencing data list was analyzed in terms of species-specific read number (SSRN), genome coverage (\%), and depth. Instead of SSRN, genus-specific read number was used for the Mycobacterium tuberculosis complex, because M. tuberculosis complex members exhibit $>99.99 \%$ genomic sequence similarity (Supply and Brosch, 2017). Probable causative microorganisms were identified with reference to the literature and when the pathogenicity was consistent with the clinical manifestations; they were verified using traditional methods (serological tests, smears, cultures, and/or polymerase chain reaction assay). Herpes simplex virus (HSV), varicella zoster virus, EpsteinBarr virus, and cytomegalovirus were considered to be mNGS false-positives in patients with non-viral infections. Common pathogens of bacterial meningitis (Streptococcus pneumoniae, Staphylococcus aureus, Haemophilus influenzae, Klebsiella pneumoniae, and Neisseria meningitidis) were considered to be mNGS false-positives in patients with non-bacterial meningitis. Aspergillus fumigatus, Aspergillus flavus, Aspergillus niger, and Aspergillus terreus (Supply and Brosch, 2017) were considered to be mNGS false-positives in patients who did not have CNS aspergillosis.

\section{Statistical Analysis}

Continuous data were considered to be nonparametric. Quantitative variables are expressed as medians (ranges) and qualitative variables are expressed as percentages. We drew receiver operating characteristic curves to compare diagnostic tests; test accuracies were represented by the area under the curve (AUC), such that a larger area implied a better test. Data 


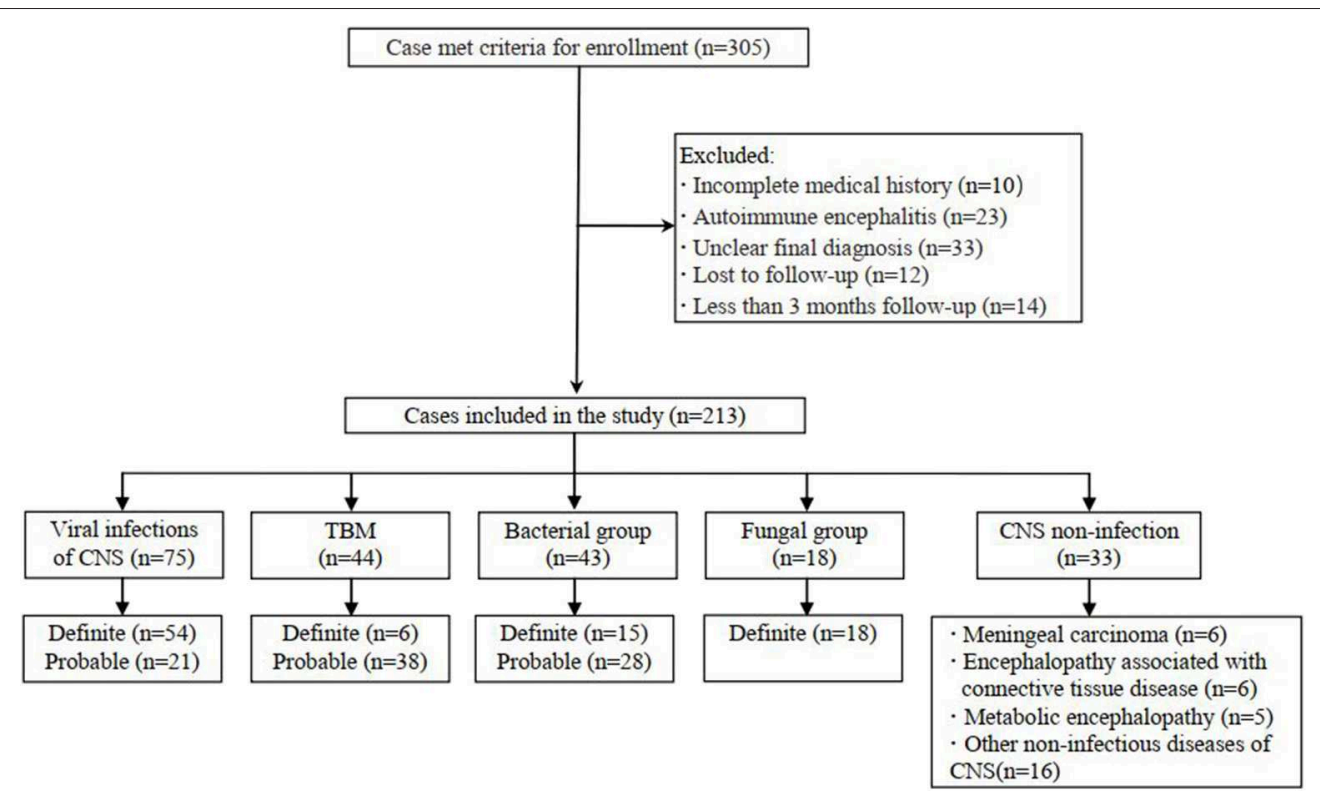

FIGURE 1 | Flowchart of study participants.

processing was performed using SPSS Statistics version 21.0 (IBM Corp., Armonk, NY, USA).

\section{RESULTS}

\section{Patient Characteristics}

In total, 213 patients treated in six hospitals were finally enrolled, including 75 with presumed viral encephalitis and/or meningitis, 44 with presumed TBM, 43 with presumed bacterial meningitis, 18 with fungal meningitis, and 33 with presumed noninfectious CNS diseases (Figure 1). All patients with infectious encephalitis and/or meningitis were screened by a single investigator (X.-W. X.) to determine diagnostic classification. Most patients $(178 / 213,83.57 \%)$ were treated in the First Medical Center of the PLA General Hospital. Non-infectious CNS conditions included meningeal carcinoma (6/33, 18\%), encephalopathy associated with connective tissue disease $(6 / 33$, $18.18 \%)$, metabolic encephalopathy (5/33, 15.15\%), and other non-infectious diseases $(16 / 33,48.48 \%)$. The clinical data are summarized in Table 1.

\section{Use of mNGS to Diagnose Viral Encephalitis and/or Meningitis}

Seventy-five patients with viral encephalitis and/or meningitis (54 definite and 21 probable) were enrolled. An mNGS result was considered positive if the CSF sample exhibited SSRNs $\geq 1$, $2,3,5$, or 10; the diagnostic probability consistencies for viral encephalitis and/or meningitis were 32, 30.7, 29.3, 26.7, and $22.7 \%$, respectively. The negative consistency rates were 84.8 , $89.1,89.9,89.9$, and $92.8 \%$, respectively; the total consistency rates were $66.2,68.5,68.5,67.6$, and $68.1 \%$, respectively. The AUCs for the five positive mNGS criteria were 0.584, 0.599, 0.596, 0.583 , and 0.577 , respectively. When an SSRN $\geq 2$ was considered positive, the corresponding AUC (0.599) was relatively larger than when other SSRNs were considered positive. For the 54 patients with definite viral encephalitis and/or meningitis, if an SSRN $\geq 2$ was considered positive, the AUC was largest (0.659, $95 \%$ confidence interval $[\mathrm{CI}]=0.566-0.751$; Figure $2 \mathrm{~A}$ ); the positive, negative, and total consistency rates were $42.6,89.1$, and $76.0 \%$, respectively (Table 2, Table S1).

\section{Use of mNGS to Diagnose TBM}

Forty-four patients were diagnosed with TBM (six definite and 38 probable). An mNGS result was considered positive if the CSF samples exhibited genus-specific read numbers $\geq 1,2,3,5$, and 10 ; the associated positive consistency rates were $27.3,20.5,18.2$, 13.6 , and $6.8 \%$, respectively. The negative consistency rates were $96.4,97.6,98.2,99.4$, and $100 \%$, respectively; the total consistency rates were $82.2,81.7,81.7,81.2$, and $80.8 \%$, respectively. When a genus-specific read number $\geq 1$ was considered positive, the AUC $(0.619,95 \% \mathrm{CI}=0.516-0.721)$ was largest. Among the six patients with definite TBM (including five who were positive on Xpert MTB/RIF and one who was positive on acid-fast staining of nerve tissue), the sensitivity and specificity of mNGS were $66.67 \%$ (4/6) and 96.45\% (163/169), respectively. Among the 44 patients with presumed TBM (six definite and 39 probable), the CSF Xpert MTB/RIF positivity rate was $16.13 \%(5 / 31)$ and the mNGS positivity rate was $27.27 \%(12 / 44)$ (Table S2); the combined mNGS and Xpert MTB/RIF positivity rate was 29.55\% (13/44) (Figure 2B1). Unfortunately, acid-fast bacillus staining and $M$. tuberculosis culture results were negative for all CSF samples.

\section{Use of mNGS to Diagnose Bacterial Meningitis}

Forty-three patients were diagnosed with bacterial meningitis (15 definite and 28 probable). An mNGS result was considered 
TABLE 1 | Demographic and clinical characteristics of the 213 patients.

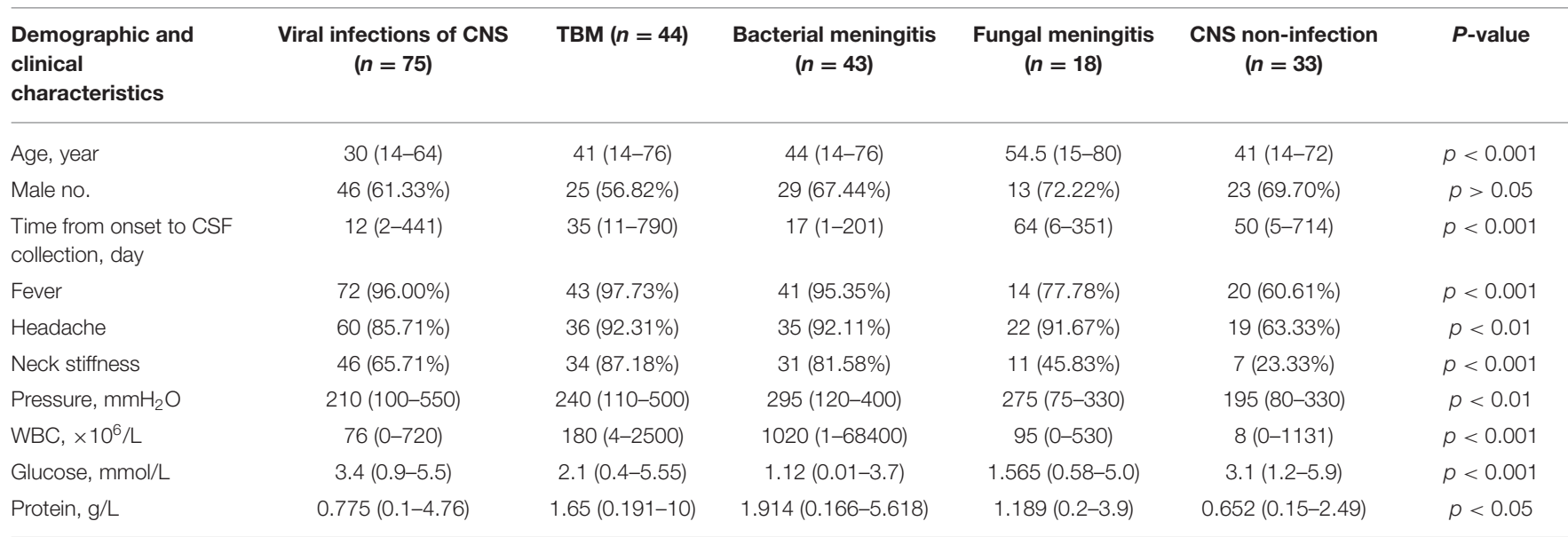

CSF, cerebrospinal fluid; CNS, central nervous system; WBC, white blood cell; TBM, tuberculous meningitis.

A

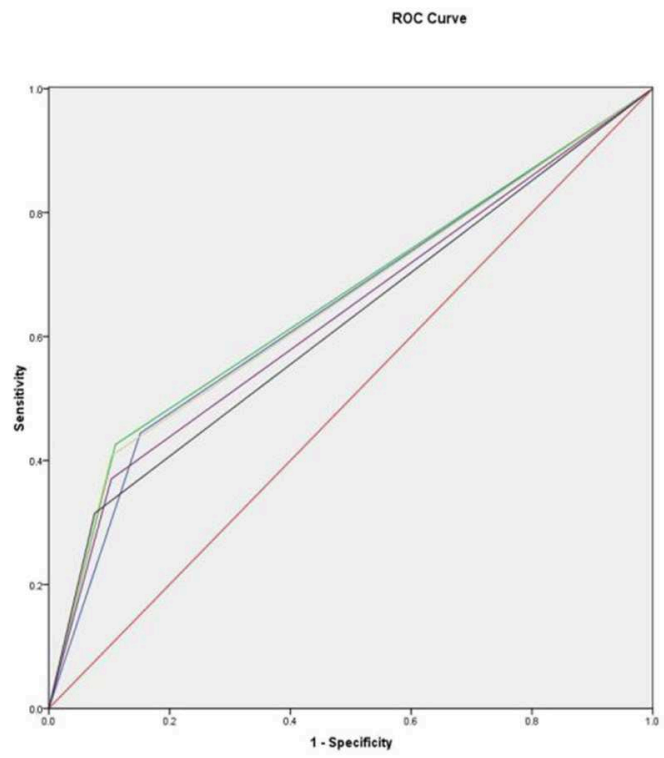

B

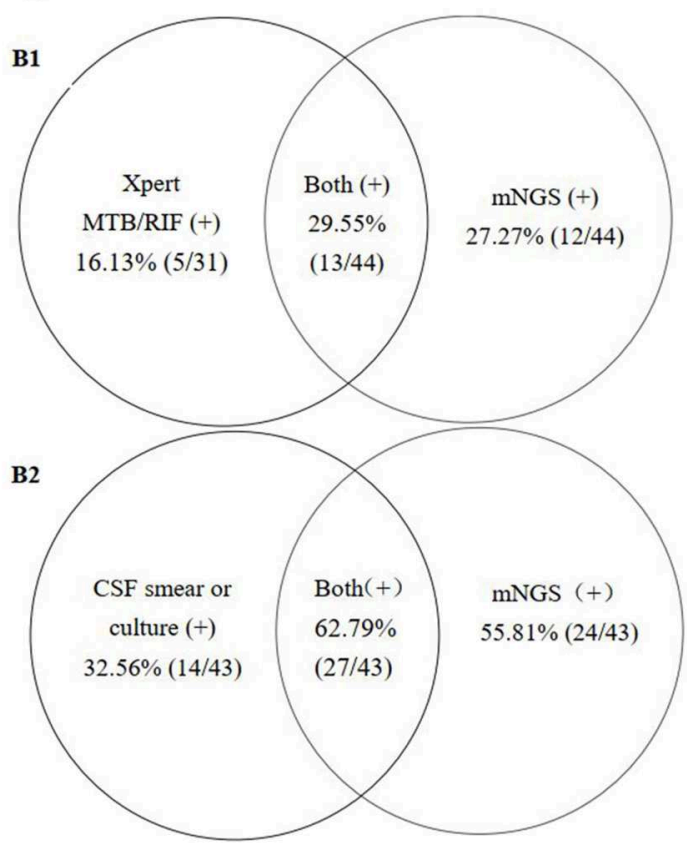

FIGURE 2 | The positive rates of mNGS combined with conventional gold microbiologic testing. (A) Receiver operating characteristic (ROC) curves for diagnosing defined viral encephalitis by mNGS. (B) Comparison of pathogens detected by mNGS compared to conventional gold standard.

positive if the SSRNs were $\geq 2,3,5,10$, and 15 ; the positive consistency rates were $62.8,62.8,55.8,55.8$, and $51.2 \%$, respectively. The negative consistency rates were $82.9,87.6,95.9$, 95.9, and 95.9\%, respectively; total consistency rates were 78.9 , $82.6,87.8,87.8$, and $86.9 \%$, respectively. The AUCs for the five positive mNGS criteria were $0.729,0.752,0.758,0.758$, and 0.735 , respectively. When SSRNs $\geq 5$ or 10 were considered positive, the AUC $(0.758,95 \%$ CI $=0.663-0.854)$ was largest. The sensitivity, specificity, positive predictive value, and negative predictive value of mNGS in the diagnosis of 15 patients with definite bacterial meningitis were $73.3,95.9,61.1$, and $97.6 \%$, respectively
(Table S3) and the AUC was largest $(0.846,95 \%$ CI $=0.711-$ $0.981)$. In brief, among the 43 patients with presumed bacterial meningitis (15 definite and 28 probable), mNGS identified a bacterial pathogen in $24(55.8 \%, 24 / 43)$; conversely, the CSF combined Gram stain/culture-positive rate was only $32.56 \%$ (14/43). Combination of the two methods increased the positive rate to $62.79 \%(27 / 43)$ (Figure 2B2). In the 24 patients with positive mNGS results (SSRNs $\geq 5$ or 10 ), the top three pathogens were S. pneumoniae $(41.7 \%, 10 / 24)$, K. pneumoniae $(12.5 \%$, $3 / 24)$, and Listeria monocytogenes (8.3\%, 2/24). Of note, among the patients with non-bacterial meningitis, $4.1 \%(7 / 170)$ were 
TABLE 2 | Comparison of the diagnostic efficacy of five positive mNGS criteria for viral encephalitis and/or meningitis (Definite, $n=54$ ).

\begin{tabular}{ccccccc}
\hline SSRN & $\begin{array}{c}\text { Positive } \\
\text { consistent } \\
\text { rate }\end{array}$ & $\begin{array}{c}\text { Negative } \\
\text { consistent } \\
\text { rate }\end{array}$ & $\begin{array}{c}\text { Total } \\
\text { consistent } \\
\text { rate }\end{array}$ & AUC & SE & 95\% CI \\
\hline 1 & 0.444 & 0.848 & 0.734 & 0.646 & 0.047 & $0.554-0.738$ \\
$\geq 2$ & 0.426 & 0.891 & 0.760 & 0.659 & 0.047 & $0.566-0.751$ \\
$\geq 3$ & 0.407 & 0.899 & 0.760 & 0.653 & 0.047 & $0.560-0.746$ \\
$\geq 5$ & 0.370 & 0.899 & 0.760 & 0.634 & 0.048 & $0.541-0.728$ \\
$\geq 10$ & 0.315 & 0.928 & 0.755 & 0.621 & 0.048 & $0.527-0.715$
\end{tabular}

SSRN, species-specific read number; AUC, area under the curve; SE, standard error; $\mathrm{Cl}$, confidence interval.

false-positive on mNGS for S. aureus $(57.1 \%, 4 / 7)$, S. pneumoniae $(28.6 \%, 2 / 7)$, and $H$. influenzae $(14.3 \%, 1 / 7)$.

\section{Use of mNGS to Diagnose Fungal Meningitis}

Among the 13 patients with definite cryptococcal meningitis, the first-time-positive rates of CSF India ink staining and fungal culture were 0 and $38.4 \%$, respectively. After multiple stainings/cultures, the positive rate of the above two methods were $84.62 \%$. Cryptococcal antigen was detected in all (12/12) CSF samples. When an SSRN $\geq 2$ was considered mNGSpositive, the sensitivity, specificity, positive predictive value, and negative predictive value were $76.92 \%$ (10/13), 99.52\% (1/207), 90.91\% (10/11), and 98.56\% (206/209), respectively (Table S4). In addition, among the 13 patients, five were co-infected with Cryptococcus neoformans sensu lato and Cryptococcus gattii sensu lato, as has been described in detail elsewhere (Xing et al., 2019). Among the five patients with confirmed cerebral aspergillosis, when an SSRN $\geq 2$ was considered mNGS-positive, the sensitivity, specificity, positive predictive value, and negative predictive value were $80 \%$ (4/5), 79.3\% (165/208), 8.5\% (4/47), and $99.40 \%$ (165/166), respectively (Table S5).

\section{Use of mNGS to Diagnose Four Types of CNS Infection}

The mNGS-positive rate for diagnosis of the four types of definite or probable infectious encephalitis and meningitis, including viral encephalitis and/or meningitis, TBM, bacterial meningitis, and fungal meningitis, was 40.6\% (73/180) (Table 3). The positivity rate for the four types of definite CNS infectious diseases was $57.0 \%(52 / 93)$.

\section{DISCUSSION}

We explored whether mNGS, combined with conventional microbiological testing, aided in the diagnosis of infectious encephalitis and meningitis. All patients met the criteria for definite or probable diagnosis of CNS infection. We made two important observations. First, different CNS infections were associated with different positive diagnostic criteria because of variations in genomic sequences and lifestyles. Second, mNGS is more effective for detection of CNS infections, compared to conventional methods (Figure 2B). Furthermore, mNGS combined with conventional microbiological testing improved detection of CNS infections.

Among the 75 patients with presumed encephalitis and/or meningitis (54 definite and 21 probable), all pathogens identified via mNGS were DNA viruses (mostly HSV-1, HSV-2, and varicella zoster virus), consistent with the findings of other studies (Steiner et al., 2007; Tyler, 2018). However, tumors and HSV infection can both trigger autoimmune encephalitis (Pruss et al., 2012). Thus, in the viral encephalitis and/or meningitis group, all patients with suspected autoimmune disorders were excluded. Identification of specific viral encephalitis/meningitis pathogens is difficult; the gold standard pathogen-specific polymerase chain reaction assays fail to identify many viral families that infect the CNS (Koyuncu et al., 2013). In theory, mNGS is useful for detection of all pathogens in clinical samples. We found that mNGS did not significantly predict viral encephalitis and/or meningitis $(0.5<$ AUC $<0.7)$, possibly attributable in part to the absence of RNA detection. RNA viruses, such as enteroviruses and Japanese encephalitis virus, are common causes of viral encephalitis and meningitis (Le et al., 2010; Ai et al., 2017). Thus, DNA/RNA co-extraction methods must be improved and DNA and RNA sequenced simultaneously to improve virus detection rates.

There were 10 million new cases of tuberculosis worldwide in 2017, of which 558,000 were rifampicin-resistant. Although only $1 \%$ of all tuberculosis infections involve the CNS, TBM is the most serious manifestation of TB; over $50 \%$ of affected patients become disabled or die (Thwaites et al., 2013). However, early accurate diagnosis of TBM remains challenging, which greatly affects patient outcomes. The pathogen complex is termed M. tuberculosis complex; M. tuberculosis, Mycobacterium africanum, Mycobacterium bovis, and Mycobacterium canettii were all detected in the present study. Thus, mNGS is of great utility in terms of TBM diagnosis when at least one specific read is matched to the $M$. tuberculosis complex, consistent with the findings of previous studies (Miao et al., 2018; Wang et al., 2019). Although the diagnostic utility of mNGS for TBM is not high (0.619), mNGS is undoubtedly very useful given the current diagnostic predicament. Of note is that the specificity of mNGS in the diagnosis of tuberculous meningitis is $96.4 \%$, which allows a negative mNGS test to be used as one of the diagnostic methods to exclude TBM.

Bacterial meningitis is a considerable burden worldwide, associated with high-level morbidity, mortality, and disability (Chaudhuri, 2004). S. pneumoniae, N. meningitidis, $H$. influenzae, and L. monocytogenes are the most common pathogens of community-acquired suppurative meningitis (Bijlsma et al., 2016). We found that $S$. pneumoniae was the principal causative pathogen in this study. When SSRNs $\geq 5$ or 10 were considered positive, mNGS performance was optimal in terms of bacterial meningitis diagnosis $(\mathrm{AUC}=0.846)$; $\mathrm{mNGS}$ may be very useful in this context. However, S. aureus is a very common pathogen that may cause a false-positive result if present in CSF.

We found that that the diagnostic utility of mNGS for cryptococcal meningitis was poorer than the utility of traditional methods (India ink staining, culture, and cryptococcal antigen detection). The thick cryptococcal capsule may not have been 
TABLE 3 | Positive mNGS results of the four types of CNS infection (73/180).

\begin{tabular}{|c|c|c|c|c|}
\hline \multicolumn{4}{|c|}{ Results of mNGS } & Confirmatory data \\
\hline \multicolumn{5}{|c|}{ Viral encephalitis and/or meningitis $(n=23)$, SSRN $\geq 2$} \\
\hline Herpes simplex virus $1(n=9)$ & $2,764(2-27639)$ & $57.9066(0.0412-91)$ & $\begin{array}{c}2.99 \\
(1-7.5)\end{array}$ & $\begin{array}{l}\text { PCR }(n=5), \text { Positive HSV antibody (CLIA) } \\
(n=9)\end{array}$ \\
\hline Herpes simplex virus $2(n=1)$ & 4,311 & 90 & 6.5 & Positive HSV antibody (CLIA) \\
\hline Epstein-Barr virus $(n=6)$ & $124.5(4-3807)$ & $\begin{array}{l}6.8258 \\
(0.1022-71.6965)\end{array}$ & $\begin{array}{c}1 \\
(1-2.39)\end{array}$ & $\begin{array}{l}\text { PCR }(n=3) ; \text { Neuropathology }(n=1 \\
\text { DLBCL); Positive EBV antibody }(n=4)\end{array}$ \\
\hline Cytomegalovirus $(n=1)$ & 4 & 0.081 & 1 & CSF cytomegalovirus lgG (122.0 U/mL) \\
\hline Human adenovirus B1 $(n=1)$ & 2,467 & 62.309 & 9.84 & Clinical evidence \\
\hline \multicolumn{5}{|l|}{ Tuberculous meningitis $(n=12)$, GSRN $\geq 1$} \\
\hline Mycobacterium tuberculosis complex & $4(1-1046)$ & ND & ND & $\begin{array}{l}\text { Xpert MTB/RIF }(n=3) \text {; A. TB or } \\
\text { T-SPOT.TB }(n=4) \text {; Tuberculosis antibody } \\
(n=1) \text {; Clinical evidence }(n=4)\end{array}$ \\
\hline \multicolumn{5}{|c|}{ Bacterial meningitis $(n=24)$, SSRN $\geq 5$ or 10} \\
\hline Streptococcus pyogenes $(n=1)$ & 453 & 3.7 & 1 & Smear \\
\hline Streptococcus intermedius $(n=1)$ & 592 & 5.2651 & 1 & Clinical evidence \\
\hline Klebsiella pneumoniae $(n=3)$ & $15(12-70)$ & $\begin{array}{l}0.0241 \\
(0.0127-0.2979)\end{array}$ & $\begin{array}{c}1 \\
(1-1.59)\end{array}$ & Culture $(n=3)$ \\
\hline Listeria monocytogenes $(n=2)$ & $43.5(36-51)$ & $\begin{array}{l}0.1167 \\
(0.0418-0.1915)\end{array}$ & $\begin{array}{c}1.01 \\
(1-1.02)\end{array}$ & Culture $(n=2)$ \\
\hline Nocardia farcinica $(n=1)$ & 277 & 0.2631 & 1.16 & Clinical evidence \\
\hline Brucella $(n=1)$ & 18 (GSRN) & ND & ND & $\mathrm{RBPT}(+)$ and SAT $(+)$ \\
\hline Stenotrophomonas maltophilia $(n=1)$ & 288 & 0.7879 & 1 & Clinical evidence \\
\hline Haemophilus influenzae $(n=1)$ & 12 & 0.1478 & 1 & Clinical evidence \\
\hline Aspergillus $(n=4)$ & 6 (3-9) (GSRN) & ND & ND & Histopathology \\
\hline
\end{tabular}

CLIA, chemiluminescence immunoassay; CNS, central nerve system; DLBCL, diffused large b-cell lymphoma; GSRN, genus-specific reads number; mNGS, metagenomic nextgeneration sequencing; PCR, polymerase chain reaction; RBPT, rose bengal plate agglutination test; SAT, serum agglutination test; s.l., sensu lato; SSRN, species-specific read number.

Positive HSV antibody: a 4-fold or more increase in CSF virus-specific antibody titer, or CSF virus-specific IgM antibody.

Positive EBV antibody: VCA (viral capsid antigens)-IgG $\geq 1: 640$, EA (early antigens)-IgG $\geq 1: 160$, or EBNA (EB nuclear antigens) $\geq 1: 2$; [Proposed Guidelines for Diagnosing Chronic Active Epstein-Barr Virus Infection].

Clinical evidence: patient's history, clinical presentation, imaging finding, routine laboratory CSF results and response to antibiotic treatment.

adequately breached, and DNA could not exit. Cell wall breakage must be improved. We found that five patients had been infected with C. neoformans s.l. and C. gattii s.l., and thus required prolonged courses of antifungal therapy (Perfect et al., 2010; Chen et al., 2013). mNGS of CSF can be used to identify Cryptococcus species, facilitating cryptococcal meningitis diagnosis and management. It is difficult to identify the source of cerebral aspergillosis; histopathology is the gold standard diagnostic method (Bao et al., 2014). Although there were few patients with cerebral aspergillosis in this study, we presume that mNGS may serve as a future, frontline diagnostic test for cerebral aspergillosis because of the non-invasive nature of mNGS.
We analyzed the diagnostic utility of mNGS in patients with four common forms of infectious encephalitis and/or meningitis; we speculate that mNGS could be used in diagnosis of all infectious CNS diseases, including those caused by rare or new pathogens. mNGS was better than conventional methods in the diagnosis of infectious encephalitis and/or meningitis, especially in terms of species identification. However, this novel approach should be used in conjunction with conventional microbiological testing. The data should be interpreted differently, depending on the pathogen involved.

Our study had some strength. The principal strength was that we divided eligible patients into groups with 
different CNS infections, rather than pooling all patients with CNS infections. The mNGS results were interpreted with reference to the different types of infections. Moreover, the study was prospective in nature. However, there were also several limitations in this study. Firstly, RNA-Seq data were not tested in parallel with DNA sequencing, which might provide valuable complementary information. Furthermore, because DNA extraction efficiency is critical in terms of mNGS results, a comparison of the extraction efficiencies of the various kits must be performed in future studies. Finally, our sample size was relatively small, especially after stratification of patients according to the types of infections.

As a novel form of microbiological testing, mNGS affords certain advantages over traditional tests when identifying pathogens causing infectious encephalitis and meningitis. The new technology exhibits great potential. Careful attention is needed with respect to DNA and RNA co-extraction methods, extraction efficiency, differentiation of colonization from infection, and method standardization (Kennedy et al., 2017; Simner et al., 2018).

\section{DATA AVAILABILITY STATEMENT}

The raw data supporting the conclusions of this article will be made available by the authors, without undue reservation, to any qualified researcher. Some of sequencing data is available at: http://www.ncbi.nlm.nih.gov/bioproject/PRJNA544609.

\section{REFERENCES}

Ai, J., Xie, Z., Liu, G., Chen, Z., Yang, Y., and Li, Y., et al. (2017). Etiology and prognosis of acute viral encephalitis and meningitis in Chinese children: a multicentre prospective study. BMC Infect. Dis. 17:494. doi: 10.1186/s12879-017-2572-9

Bao, Z. S., You, G., Li, W. B., and Jiang, T. (2014). A single aspergillus fumigatus intracranial abscess in an immunocompetent patient with parietal lobe tumorectomy. World J. Surg. Oncol. 12:181. doi: 10.1186/1477-781912-181

Bijlsma, M. W., Brouwer, M. C., Kasanmoentalib, E. S., Kloek, A. T., Lucas, M. J., Tanck, M. W., et al. (2016). Community-acquired bacterial meningitis in adults in the Netherlands, 2006-14: a prospective cohort study. Lancet Infect. Dis. 16, 339-347. doi: 10.1016/S1473-3099(15)00430-2

Chaudhuri, A. (2004). Adjunctive dexamethasone treatment in acute bacterial meningitis. Lancet Neurol. 3, 54-62. doi: 10.1016/S1474-4422(03)00623-9

Chen, S. C., Korman, T. M., Slavin, M. A., Marriott, D., Byth, K., Bak, N., et al. (2013). Antifungal therapy and management of complications of cryptococcosis due to Cryptococcus gattii. Clin. Infect. Dis. 57, 543-551. doi: $10.1093 / \mathrm{cid} / \mathrm{cit} 341$

Forbes, J. D., Knox, N. C., Ronholm, J., Pagotto, F., and Reimer, A. (2017). Metagenomics: the next culture-independent game changer. Front. Microbiol. 8:1069. doi: 10.3389/fmicb.2017.01069

Glaser, C. A., Honarmand, S., Anderson, L. J., Schnurr, D. P., Forghani, B., Cossen, C. K., et al. (2006). Beyond viruses: clinical profiles and etiologies associated with encephalitis. Clin. Infect. Dis. 43, 1565-1577. doi: 10.1086/5 09330

Goldberg, B., Sichtig, H., Geyer, C., Ledeboer, N., and Weinstock, G. M. (2015). Making the leap from research laboratory to clinic: challenges and opportunities for next-generation sequencing in infectious disease diagnostics. MBio 6, e01888-e01815. doi: 10.1128/mBio.01888-15

\section{ETHICS STATEMENT}

This study was approved by the Ethics Committee of the Chinese PLA General Hospital (approval no. S2018-198-01). Written informed consent was obtained from all patients or their legal representatives.

\section{AUTHOR CONTRIBUTIONS}

J-TZ designed the study. S-YY organized and delivered the expert meeting, which was attended by all the authors. Y-BM, M-WH, G-EY, WW, X-KQ, X-YC, LW, X-LW, Y-HH, JD, H-FW, R-FW, and FY contributed to acquisition of clinical data. X-WX conducted analyses and wrote the manuscript. All of the authors read and approved the final article.

\section{ACKNOWLEDGMENTS}

We thank all patients and their families for participation in the study, and we thank the Tianjin Medical Laboratory, BGITianjin, Tianjin, China, for sharing their mNGS procedure and providing a sequencing data list for this study.

\section{SUPPLEMENTARY MATERIAL}

The Supplementary Material for this article can be found online at: https://www.frontiersin.org/articles/10.3389/fcimb. 2020.00088/full\#supplementary-material

Guan, H., Shen, A., Lv, X., Yang, X., Ren, H., Zhao, Y., et al. (2016). Detection of virus in CSF from the cases with meningoencephalitis by next-generation sequencing. J. Neurovirol. 22, 240-245. doi: 10.1007/s13365-015-0390-7

Jeffery, K. J., Read, S. J., Peto, T. E., Mayon-White, R. T., and Bangham, C. R. (1997). Diagnosis of viral infections of the central nervous system: clinical interpretation of PCR results. Lancet 349, 313-317. doi: 10.1016/S0140-6736(96)08107-X

Kennedy, P. G. E., Quan, P. L., and Lipkin, W. I. (2017). Viral encephalitis of unknown cause: current perspective and recent advances. Viruses 9:138. doi: 10.3390/v9060138

Koyuncu, O. O., Hogue, I. B., and Enquist, L. W. (2013). Virus infections in the nervous system. Cell Host Microbe 13, 379-393. doi: 10.1016/j.chom.2013.03.010

Le, V. T., Phan, T. Q., Do, Q. H., Nguyen, B. H., Lam, Q. B., Bach, V., et al. (2010). Viral etiology of encephalitis in children in southern Vietnam: results of a one-year prospective descriptive study. PLoS Negl. Trop. Dis. 4:e854. doi: 10.1371/journal.pntd.0000854

Marais, S., Thwaites, G., Schoeman, J. F., Torok, M. E., Misra, U. K., Prasad, K., et al. (2010). Tuberculous meningitis: a uniform case definition for use in clinical research. Lancet Infect. Dis. 10, 803-812. doi: 10.1016/S1473-3099(10)70138-9

Miao, Q., Ma, Y., Wang, Q., Pan, J., Zhang, Y., Jin, W., et al. (2018). Microbiological diagnostic performance of metagenomic next-generation sequencing when applied to clinical practice. Clin. Infect. Dis. 67, S231-S240. doi: $10.1093 /$ cid/ciy693

Mudaliar, A. V., Kashyap, R. S., Purohit, H. J., Taori, G. M., and Daginawala, H. F. (2006). Detection of $65 \mathrm{kD}$ heat shock protein in cerebrospinal fluid of tuberculous meningitis patients. BMC Neurol. 6:34. doi: 10.1186/1471-2377-6-34

Perfect, J. R., Dismukes, W. E., Dromer, F., Goldman, D. L., Graybill, J. R., Hamill, R. J., et al. (2010). Clinical practice guidelines for the management of 
cryptococcal disease: 2010 update by the infectious diseases society of america. Clin. Infect. Dis. 50, 291-322. doi: 10.1086/649858

Pruss, H., Finke, C., Holtje, M., Hofmann, J., Klingbeil, C., Probst, C., et al. (2012). $\mathrm{N}$-methyl-D-aspartate receptor antibodies in herpes simplex encephalitis. Ann. Neurol. 72, 902-911. doi: 10.1002/ana.23689

Simner, P. J., Miller, S., and Carroll, K. C. (2018). Understanding the promises and hurdles of metagenomic next-generation sequencing as a diagnostic tool for infectious diseases. Clin. Infect. Dis. 66, 778-788. doi: 10.1093/cid/cix881

Steiner, I., Kennedy, P. G., and Pachner, A. R. (2007). The neurotropic herpes viruses: herpes simplex and varicella-zoster. Lancet Neurol. 6, 1015-1028. doi: 10.1016/S1474-4422(07)70267-3

Supply, P., and Brosch, R. (2017). The biology and epidemiology of mycobacterium canettii. Adv. Exp. Med. Biol. 1019, 27-41. doi: 10.1007/978-3-319-64371-7_2

Thwaites, G. E., Van Toorn, R., and Schoeman, J. (2013). Tuberculous meningitis: more questions, still too few answers. Lancet Neurol. 12, 999-1010. doi: 10.1016/S1474-4422(13)70168-6

Tyler, K. L. (2018). Acute viral encephalitis. N. Engl. J. Med. 379, 557-566. doi: 10.1056/NEJMra1708714

Venkatesan, A., Tunkel, A. R., Bloch, K. C., Lauring, A. S., Sejvar, J., Bitnun, A., et al. (2013). Case definitions, diagnostic algorithms, and priorities in encephalitis: consensus statement of the international encephalitis consortium. Clin. Infect. Dis. 57, 1114-1128. doi: 10.1093/cid/cit458

Wang, S., Chen, Y., Wang, D., Wu, Y., Zhao, D., Zhang, J., et al. (2019). The feasibility of metagenomic next-generation sequencing to identify pathogens causing tuberculous meningitis in cerebrospinal fluid. Front. Microbiol. 10:1993. doi: $10.3389 /$ fmicb.2019.01993

Wilson, M. R., Naccache, S. N., Samayoa, E., Biagtan, M., Bashir, H., Yu, G., et al. (2014). Actionable diagnosis of neuroleptospirosis by next-generation sequencing. N. Engl. J. Med. 370, 2408-2417. doi: 10.1056/NEJMoal401268
Wilson, M. R., Sample, H. A., Zorn, K. C., Arevalo, S., Yu, G., Neuhaus, J., et al. (2019). Clinical metagenomic sequencing for diagnosis of meningitis and encephalitis. N. Engl. J. Med. 380, 2327-2340. doi: 10.1056/NEJMoa18 03396

Xing, X. W., Zhang, J. T., Ma, Y. B., Chen, X. Y., Wu, L., Wang, X. L., et al. (2018). Evaluation of next-generation sequencing for the diagnosis of infections of the central nervous system caused by the neurotropic herpes viruses: a pilot study. Eur. Neurol. 80, 283-288. doi: 10.1159/000 497319

Xing, X. W., Zhang, J. T., Ma, Y. B., Zheng, N., Yang, F., and Yu, S. Y. (2019). Apparent performance of metagenomic next-generation sequencing in the diagnosis of cryptococcal meningitis: a descriptive study. J. Med. Microbiol. 68, 1204-1210. doi: 10.1099/jmm.0.000994

Yao, M., Zhou, J., Zhu, Y., Zhang, Y., Lv, X., Sun, R., et al. (2016). Detection of listeria monocytogenes in csf from three patients with meningoencephalitis by next-generation sequencing. J. Clin. Neurol. 12, 446-451. doi: 10.3988/jen.2016.12.4.446

Conflict of Interest: The authors declare that the research was conducted in the absence of any commercial or financial relationships that could be construed as a potential conflict of interest.

Copyright $\odot 2020$ Xing, Zhang, Ma, He, Yao, Wang, Qi, Chen, Wu, Wang, Huang, $D u$, Wang, Wang, Yang and Yu. This is an open-access article distributed under the terms of the Creative Commons Attribution License (CC BY). The use, distribution or reproduction in other forums is permitted, provided the original author(s) and the copyright owner(s) are credited and that the original publication in this journal is cited, in accordance with accepted academic practice. No use, distribution or reproduction is permitted which does not comply with these terms. 